\title{
Heavy-quark effects on cold quark matter and self-bound stars
}

\author{
José C. Jiménez ${ }^{1, *}$ and Eduardo S. Fraga ${ }^{2, * *}$ \\ ${ }^{1}$ Instituto de Física, Universidade de São Paulo, \\ Rua do Matão, 1371, Butantã, 05508-090, São Paulo, SP, Brazil \\ ${ }^{2}$ Instituto de Física, Universidade Federal do Rio de Janeiro, \\ Caixa Postal 68528, 21941-972, Rio de Janeiro, RJ, Brazil
}

\begin{abstract}
The heavy-quark effects on the equation of state for cold and dense quark matter are obtained from perturbative QCD, yielding observables parametrized only by the renormalization scale. In particular, we investigate the thermodynamics of charm quark matter under the constraints of $\beta$ equilibrium and electric charge neutrality in a region of densities where perturbative QCD is, in principle, much more reliable. Finally, we analyze the stability of charm stars, which might be realized as a new branch of ultradense hybrid compact stars, and find that such self-bound stars are unstable under radial oscillations.
\end{abstract}

\section{Introduction}

The presence of heavy flavors in cold quark matter might have non-trivial effects in matter subject to extreme conditions of density. For instance, the particular case of charm quarks immeaditely lead us to consider the possibility of charm stars, i.e. self-bound strange stars with some amount of charm quarks at their cores. However, although usual constraints forbid their existence [1, 2], they might still exist in ultradense hybrid neutron stars. Besides, perturbative quantum chromodynamics (pQCD) can be used much more confidently at these very high densities [3] when building the equation of state $(\mathrm{EoS})$ for charm matter ${ }^{1}$ in electroweak equilibrium. In this proceeding, we investigate cold quark matter with heavy quarks using in-medium pQCD. Specifically, we build our framework using the formalism developed in Ref. [6] for $N_{f}=N_{l}+1$ flavors, i.e. $N_{l}$ is the number of massless quarks and " 1 " the massive flavor, to include any number of massive flavors and determine the associated EoS. As an application of our framework, we discuss charm matter in $\beta$ equilibrium and also being electrically neutral. Interestingly, this allow us to discuss the existence charm quark stars from the pQCD viewpoint. In the past, these exotic stars were investigated only within the bag model being unstable against radial oscillations [7]. By applying the Gondek's method [8] for a couple of first-order differential equations for the Lagrangian radial displacements, our calculations indicate that such self-bound stars are unstable.

\footnotetext{
*e-mail: jimenez@if.usp.br

**e-mail: fraga@if.ufrj.br

${ }^{1}$ This complements advancements in PQCD [4] and lattice QCD at high temperatures [5].
} 


\section{Cold and dense PQCD with heavy quarks}

The calculation of the EoS for cold quark matter within pQCD has a long history [3]. The state-of-the-art three loop result of Ref. [6] includes renormalization-group effects of the mass and strong coupling parameters. More especifically, this framework deals with $N_{f}=N_{l}+1$ quark flavors, i.e., $N_{l}$ massless plus 1 massive, e.g. massless up and down and massive strange. The corresponding pQCD thermodynamic potential is composed by a massless contribution plus a massive term, together with the mixed vacuum-matter (VM) diagrams and the corresponding ring terms, i.e. $\Omega=\Omega^{m=0}(\vec{\mu})+\Omega^{m}(\tilde{\mu}, m)+\Omega_{\mathrm{VM}}^{x}(\vec{\mu}, m)+\Omega_{\text {ring }}(\vec{\mu}, \tilde{\mu}, m)$ with mass $m$ and $\tilde{\mu}$ corresponds to the massive flavor $\vec{\mu} \equiv\left(\mu_{1}, \ldots, \mu_{N_{l}}\right)$ is the vector chemical potential for the massless quarks. Besides, this $\Omega$ depends on a renormalization scale parameter $\bar{\Lambda}$, which is a pQCD scale and $\alpha_{s}$ is $\alpha_{s}(\bar{\Lambda})=\left(4 \pi / \beta_{0} L\right)\left(1-\left(2 \beta_{1} / \beta_{0}^{2}\right) \ln L / L\right)$ where $\beta_{0}=11-2 N_{f} / 3, \beta_{1}=51-19 N_{f} / 3$, and $L=2 \ln \left(\bar{\Lambda} / \Lambda_{\overline{\mathrm{MS}}}\right)$ with $\Lambda_{\overline{\mathrm{MS}}}$ as the $\overline{\mathrm{MS}}$ point scale. Usually the renormalization scale is defined as $\bar{\Lambda}=X \sum_{i} \mu_{i} / N_{f}$, where the sum runs over the active quark flavors, and the dimensionless parameter $X$ sits between 1 and 4 [6].

On the other hand, at high densities one expects that light quarks could be accompanied by heavy flavors. In this situation, the total number of flavors are $N_{f}=\sum_{i=1}^{N_{m}}\left(N_{l}+1\right)^{(i)}$, where $N_{m}$ is the number of massive quarks. This restrict us to add at least one massless quark for each massive flavor. Notice that further physical conditions are required in order to control when a heavy partner appears actively. In fact, such conditions affect the renormalization scale $\bar{\Lambda}$, depending on the chosen heavy quark to be added. Thus, the $\Omega$ at $O\left(\alpha_{s}^{2}\right)$ for $N_{l}$ massless and $N_{m}$ massive quarks is $\bar{\Omega}\left[N_{f}\right]=\sum_{i=1}^{N_{m}}\left\{\Omega\left[N_{l}^{(i)}\right]+\Omega\left[1^{(i)}\right]\right\}$, choosing first the massless flavors when adding a massive one, so that $\Omega\left[N_{l}^{(i)}\right] \equiv\left(\Omega^{m=0}(\vec{\mu})\right)^{(i)}$ is the massless contribution and $\Omega\left[1^{(i)}\right] \equiv\left(\Omega^{m}+\Omega_{\mathrm{VM}}^{x}+\Omega_{\text {ring }}\right)^{(i)}$ is the mixed massive contribution, where $\vec{\mu}^{(i)}=\left(\mu_{1}, \ldots, \mu_{i}\right)$ is the massless vector chemical potential, $\tilde{\mu}^{(i)}$ the massive (heavy) quark chemical potentials, and $m^{(i)}$ their corresponding masses. Here, $\Omega[\ldots]$ indicates just the implicit parameter dependence (e.g. on $N_{f}$ ), whereas $\Omega(\ldots)$ represents an explicit function dependence.

\section{Charm matter thermodynamics}

We now apply the aforementioned formalism to charm quark matter for which pQCD is more reliable. The EoS for charm matter is built as follows. Below the charm threshold, the usual strange matter conditions are the same [9]. Above the charm threshold, the reactions $u+d \leftrightarrow c+d$ yield $\mu_{c}=\mu_{u}$. Furthermore, electric neutrality is $(2 / 3) n_{u}+(2 / 3) n_{c}-$ $(1 / 3) n_{d}-(1 / 3) n_{s}-n_{e}-n_{\mu}=0$, with muons having being degenerate satisfying $\mu_{\mu}=\mu_{e}$. Then, all chemical potentials are functions of $\mu_{s}$. The strange and charm quark mass parameters (up to $\left.O\left(\alpha_{s}^{2}\right)\right)$ are [10] $m_{s}(\bar{\Lambda})=\hat{m}_{s}\left(\alpha_{s} / \pi\right)^{4 / 9}\left(1+0.895062\left(\alpha_{s} / \pi\right)+1.37143\left(\alpha_{s} / \pi\right)^{2}\right)$ and $m_{c}(\bar{\Lambda})=\hat{m}_{c}\left(\alpha_{s} / \pi\right)^{12 / 25}\left(1+1.01413\left(\alpha_{s} / \pi\right)+1.38921\left(\alpha_{s} / \pi\right)^{2}\right)$, respectively. The $\left\{\hat{m}_{q}\right\}$ are the invariant quark masses. By noting that $\alpha_{s}$ depends on $N_{f}$, this gives different values of $\Lambda_{\overline{\mathrm{MS}}}$. Choosing $\alpha_{s}\left(\bar{\Lambda}=1.5 \mathrm{GeV}, N_{f}=3,4\right)=0.336_{-0.008}^{+0.012}[11]$ gives $\Lambda_{\overline{\mathrm{MS}}}^{2+1}=343_{-12}^{+18} \mathrm{MeV}$ and $\Lambda_{\overline{\mathrm{MS}}}^{2+1+1}=290_{-12}^{+18} \mathrm{MeV}$, thus defining $\alpha_{s}^{2+1}(\bar{\Lambda})$ and $\alpha_{s}^{2+1+1}(\bar{\Lambda})$, respectively. Fixing the strange quark mass at $m_{s}\left(2 \mathrm{GeV}, N_{f}=3,4\right)=92.4(1.5) \mathrm{MeV}$ [12] give $\hat{m}_{s}^{2+1} \approx 246.2 \mathrm{MeV}$ when using $\alpha_{s}^{2+1}$, and $\hat{m}_{s}^{2+1+1} \approx 243.7 \mathrm{MeV}$ with $\alpha_{s}^{2+1+1}$. Additionally, fixing the charm quark mass at $m_{c}\left(3 \mathrm{GeV}, N_{f}=4\right)=0.9851(63) \mathrm{GeV} \equiv m_{c}^{0}[12]$, gives $\hat{m}_{c}^{2+1+1} \approx 3.0895 \mathrm{GeV}$ when using $\alpha_{s}^{2+1+1}$, being $m_{c}^{0}$ the vacuum charm mass. Furthermore, charm quarks must satisfy $\mu_{c}=\mu_{s}-\mu_{e}>m_{c}^{\text {medium }}>m_{c}^{0}$ being $m_{c}^{\text {medium }}$ the unknown in-medium charm mass and the renormalization scale parameter below and above the charm threshold is $\bar{\Lambda}=X\left(\mu_{u}+\mu_{d}+\mu_{s}+0\right) / 3$ for $\mu_{s} \lesssim m_{c}^{0}$ and $X^{*}\left(\mu_{u}+\mu_{d}+\mu_{s}+\mu_{c}\right) / 3$ for $\mu_{s} \gtrsim m_{c}^{0}$, where the 

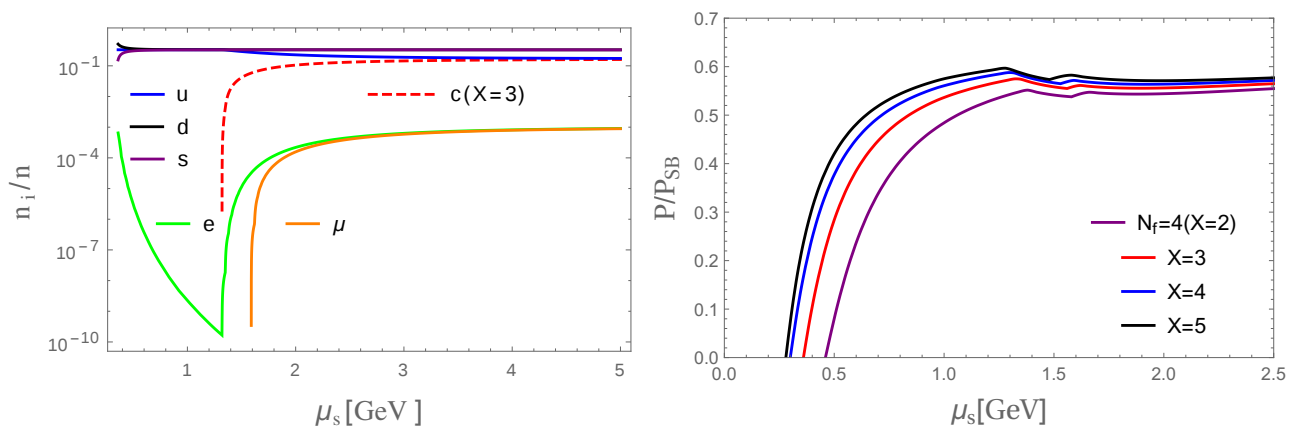

Figure 1. Left panel: Quark and lepton populations, $n_{i} / n$, present in charm matter for $X=3$. Above the charm threshold, the lepton fractions increase to ensure electric charge neutrality at high densities. Right panel: Total pressure of $N_{f}=2+1+1$ quarks plus leptons in $\beta$ equilibrium and electrically charge neutral normalized by the Stefan-Boltzmann gas with $N_{f}=4$. The case $X=5$ is shown only to verify how the EoS depends on $X$ when including one additional heavy flavor. Taken from Ref. [14].
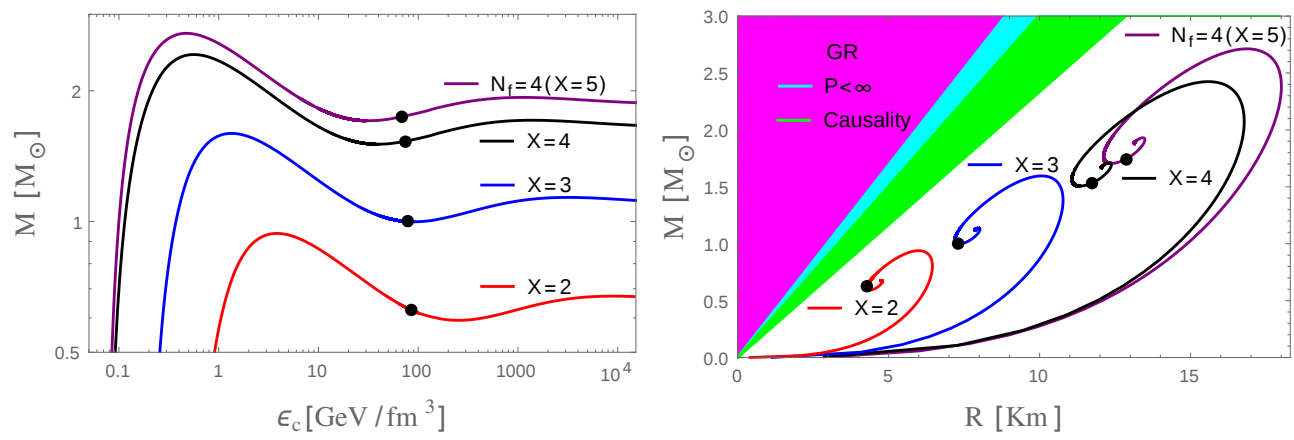

Figure 2. Left panel: Gravitational masses vs central energy densities for a system with $N_{f}=2+1+1$ quarks plus leptons. Right panel: Mass-radius diagram for the associated quark stars. The black dots indicate the appearance of the charm quarks. Stars not satisfying the general-relativistic (GR, magenta), causality (green), and finite pressure ( $P<\infty$, light blue) limits are excluded [13]. Taken from Ref. [14].

approximations in the inequalities come from noticing that before the threshold point the electron chemical potential takes its lowest value compared to the strange one, thus allowing us to make the approximation $\mu_{c} \approx \mu_{s}$. To build the EoS, we choose $N_{l}^{(1)}=1$ for the up, $N_{l}^{(2)}=1$ for the down, and $N_{m}=2$ for the strange and charm quarks, in the thermodynamic potential $\bar{\Omega}\left[N_{f}=2+1+1\right]=\left\{\Omega\left[N_{l}^{(1)}=1\right]+\Omega\left[1^{(1)}\right]\right\}+\left\{\Omega\left[N_{l}^{(2)}=1\right]+\Omega\left[1^{(2)}\right]\right\}$ so that the flavors are counted as $N_{f}=(1+1)^{(1)}+(1+1)^{(2)}=(u+c)^{(1)}+(d+s)^{(2)}$. We define the total quark number density for charm matter, for a given $X$, as $n_{q} \equiv n_{u}+n_{d}+n_{s}+n_{c}$ and the total particle density as $n=n_{q}+n_{L}$, where $n_{L}=n_{e}+n_{\mu}$. In Fig. 1, we show the behavior of the relative particle populations in $\beta$ equilibrium and electrically neutral charm quark matter in the case of $X=3$. The total pressure and energy density are obtained by keeping thermodynamic consistency systematically. This allows us to build numerically the EoS, $P=P(\epsilon, X)$. In Fig. 1 , the total pressure is displayed for charm matter normalized by a Stefan-Boltzmann gas of quarks with $N_{f}=4$ as a function of the strange quark chemical potential. Besides, the charm EoS is largely softened, generating an apparent instability which could have astrophysical effects, e.g. the possibility of charm stars. 


\section{Results and Conclusion}

The possibility of bare charm quark stars was studied in Ref. [7] using the simplest version of the bag model. Their conclusion was that charm stars would be unstable against radial perturbations. We revisit this question using our first-principle pQCD results for the EoS for charm quark matter. We also choose the range $X \geq 3$, which satisfies the Bodmer-Witten hypothesis of strange quark matter. First of all, although the structure equations ensure their relativistic hydrostatic equilibrium, they must also satisfy the condition $\partial M / \partial \epsilon_{c} \geq 0$ [9]. The maximal masses are identified where $\partial M / \partial \epsilon_{c}=0$ (see Fig. 2). However, we note that for the case $X=2$, this condition is not satisfied when charm quarks appear. On the other hand, for $X>3$, this condition is satisfied when charm quarks are present, which would correspond to stable charm stars. In Fig. 2, we show the mass-radius diagram for quark stars made of $N_{f}=2+1+1$ quarks plus electrons and muons with varying $X$. Performing the dynamical stability analysis against radial pulsations using the method of Gondek et al. [8], where amplitudes oscillate harmonically when the frequencies are such that $\operatorname{Re}\left(\omega_{\mathrm{n}}\right)>0$ and $\operatorname{Im}\left(\omega_{\mathrm{n}}\right)=0$, or increase exponentially if $\operatorname{Re}\left(\omega_{\mathrm{n}}\right) \geq 0$ and $\operatorname{Im}\left(\omega_{\mathrm{n}}\right)>0$. Besides, if $\operatorname{Im}\left(\omega_{0}\right)>0$ [and $\operatorname{Re}\left(\omega_{0}\right)=0$ ] from some value of central energy density $\epsilon_{c}$, then all the higher modes will become complex too, representing the onset of the instability. Our calculations show that for $X=3$ the amplitudes of the stellar configurations increase even in the region where charm stars are expected, thus making them dynamically unstable. Since the same behavior was obtained for larger values of $X$, one can conclude from a perturbative QCD analysis that charm stars are unstable. In this work, we have extended the pQCD $N_{f}=N_{l}+1$ formalism to include heavy quarks in the EoS for cold and dense quark matter. Their effects at low densities are non trivial through the renormalization scale parameter $\bar{\Lambda}$. After performing a dynamical analysis, it was found that charm stars are unstable. In the future, it would be interesting to study the presence of charm quarks in neutron-star mergers.

\section{Acknowledgement}

This work was partially supported by INCT-FNA (Process No. 464898/2014-5). J. C. J. acknowledges support from FAPESP (Processes No. 2020/07791-7 and No. 2018/24720-6). E. S. F. is partially supported by CAPES (Finance Code 001), CNPq, and FAPERJ.

\section{References}

[1] J. M. Lattimer and M. Prakash, arXiv:1012.3208.

[2] J. M. Lattimer, Universe 5, 159 (2019).

[3] J. I. Kapusta and C. Gale, Finite-Temperature Field Theory: Principles and Applications (Cambridge University Press, Cambridge, England, 2006).

[4] M. Laine and Y. Schroder, Phys. Rev. D 73, 085009 (2006).

[5] S. Borsanyi et al., Nature (London) 539, 69 (2016).

[6] A. Kurkela, P. Romatschke, and A. Vuorinen, Phys. Rev. D 81, 105021 (2010).

[7] C. Kettner et al., Phys. Rev. D 51, 1440 (1995).

[8] D. Gondek, P. Haensel and J. L. Zdunik, Astron. Astrophys. 325, 217 (1997)

[9] N. K. Glendenning, Compact Stars (Springer, New York, 2000).

[10] J. A. M. Vermaseren, S. A. Larin, and T. van Ritbergen, Phys. Lett. B 405, 327 (1997).

[11] A. Bazavov et al., Phys. Rev. D 90, 074038 (2014).

[12] B. Chakraborty et al., Phys. Rev. D 91, 054508 (2015).

[13] J. M. Lattimer and M. Prakash, Phys. Rep. 442, 109 (2007).

[14] J. C. Jiménez and E. S. Fraga, Phys. Rev. D 102, 034015 (2020) 